\title{
NON-STATIONARY ATTRACTORS IN THE BLINKING SYSTEMS: GHOST ATTRACTOR OF LORENZ TYPE
}

\author{
Nikita V. Barabash \\ Volga State University of Water Transport \\ Nizhny Novgorod State University \\ Russia \\ barabash@itmm.unn.ru
}

\author{
Vladimir N. Belykh \\ Volga State University of Water Transport \\ Nizhny Novgorod State University \\ Russia \\ belykh@unn.ru
}

Article history:

Received 09.11.2019, Accepted 12.12.2019

\begin{abstract}
In this paper we consider blinking systems, i.e. the systems randomly changing its structure in each sequential fixed period of time. Our goal is to find ghost attractors which arise in a blinking system being unexpectively different with respect to the attractors of composing systems. We study two blinking systems. One of them is a nonlinear rotator with switching torque and another is a blinking piecewise-linear Lorenz-type system. We prove the existence of ghost attractors in both cases for certain parameter ranges.
\end{abstract}

\section{Key words}

Blinking system, ghost attractor, stochastic switching

\section{Introduction}

The behaviour of a large number of living and technical systems can be represented as a process with instantaneous random changes in their structures. In networks of neurons and technical devices, interaction between nodes may be a subject for such changes [Mills, 1991; Parastesh et al., 2019]. The behaviour of pulse power converters can be modelled by a dynamical system, which parameters randomly and independently change their values in a piecewise-constant manner implying switching [Tse and Di Bernardo, 2002]. In literature such random and independent switching have been called blinking by analogy to blinking of an eye [Belykh et al., 2004], and systems with such o sort of switching got the name blinking systems.

Later it turned out that the blinking systems may exhibit non-trivial unexpected behaviour, which means, for example, the emergence of dynamics which is not met in each of the composing systems [Hasler et al., 2013b; Belykh et al., 2013; Barabash and Belykh, 2018a]. The attracting set corresponding to this behaviour has been called a ghost attractor.

In this talk we give examples of such systems in which we managed to find ghost attractors, and also offer a way to find them in the general case.

\section{The Blinking Model}

We consider the blinking system [Hasler et al., 2013a; Barabash and Belykh, 2018b]

$$
\dot{x}=F(x, s(t))
$$

where $x \in \mathbb{R}^{N}, s(t)$ is a random discrete scalar value equalled a constant $s_{i}, i=1,2, \ldots, M$ with the probability $p_{i}$ at each $k$-th time interval $t \in[k \tau,(k+1) \tau)$, $k \in \mathbb{Z}^{+}$. Here $\tau=$ const is a switching period.

The trajectories of the system (1) are glued at $t=k \tau$ from the trajectories of $M$ autonomous systems

$$
\dot{x}=F\left(x, s_{i}\right), \quad i=1,2, \ldots, M
$$

given at each interval $t \in[k \tau,(k+1) \tau)$ with the probability $p_{i}$. We assume that each $i$-th $N$-dimensional system (2) considered at the whole interval of time has an attractor $A_{i}$.

For sufficiently fast switching $s=s\left(\frac{t}{\tau}\right), \tau \ll 1$ there are two characteristic times in the system (1): slow time $t$ and fast time $t^{\prime}=\frac{t}{\tau}$. Expressing slow time in the blinking system (1) through fast time $t=\tau t^{\prime}$ and setting $x\left(\tau t^{\prime}\right) \rightarrow x\left(t^{\prime}\right)$ we obtain the system in the form of the averaging approach [Bogoliubov and Mitropolsky, 1966; Khas'minskii, 1966; Skorokhod et al., 2002; Kifer, 2009; Hasler et al., 2013a]

$$
\frac{d x}{d t^{\prime}}=\tau F\left(x, s\left(t^{\prime}\right)\right)
$$




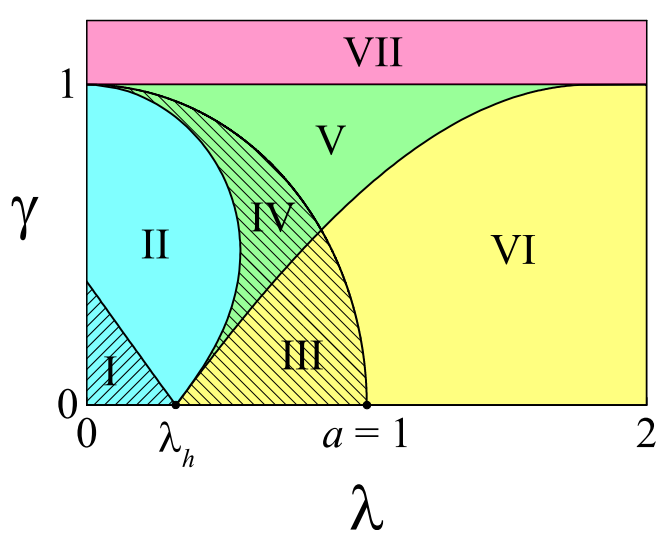

Figure 1. Bifurcation diagram of the system (6) for $a=1$. To be short we list only stable limit cycles and stable equilibria in different regions. Region I. Two stable rotating limit cycles $O_{r}^{+}$for $\dot{\varphi}>0$ and $O_{r}^{-}$for $\dot{\varphi}<0$. Region II. Globally stable rotating limit cycle $\mathrm{O}_{r}^{+}$. Region III: Globally stable oscillating limit cycle $O_{c}$. Region IV. Stable rotating limit cycle $O_{r}^{+}$and stable oscillating limit cycle $O_{c}$. Region V. Stable equilibrium $O_{e}$ and stable rotating limit cycle $\mathrm{O}_{r}^{+}$. Region VI. Globally stable equilibrium $O_{e}$. Region VII. Globally stable rotating limit cycle $O_{r}^{+}$.

where $\tau$ plays the role of a small parameter.

The autonomous $N$-dimensional averaged system associated with the system (3) has the form

$$
\frac{d x}{d t}=\mathrm{E}[F(x, s(t))]=\sum_{i=1}^{M} p_{i} F\left(x, s_{i}\right),
$$

where $\mathrm{E}[\cdot]$ is the expectation.

We assume this system to have an attractor $\bar{A}$ which serves an approximation of the blinking system dynamics for sufficiently fast switching.

The problem of the interrelation between attractors $A_{i}$, $i=1,2, \ldots, M$, and the attractor $\bar{A}$ was widely discussed in [Hasler et al., 2013b]. Here we consider a particular case of the ghost attractor [Belykh et al., 2013; Barabash and Belykh, 2018a]. We call

Definition. If the averaged system (4) is not topologically conjugated to any $i$-th autonomous system (2) and the attractor $\bar{A}$ is missing among $A_{i}$, then $\bar{A}$ we call the ghost attractor of the blinking system (1).

Non-stationary attractor $\tilde{A}$ for the blinking system (1) due to the averaging theory for infinitesimal switching period $\tau$ is close to the ghost attractor $\bar{A}$.

This definition shows the way how to find ghost attractors. For this purpose it is necessary to compare the attractors $A_{i}, i=1,2, \ldots, M$ and $\bar{A}$ and to verify whether the attractor $\bar{A}$ is different from each attractor $A_{i}$ or not.

In this paper we consider two examples of blinking systems.

The first system is the model of blinking nonlinear rotator switching between two systems having only rotational modes. We show that there exist an interval of switching period $0<\tau<\tau^{*}$ for which the blinking nonlinear rotator has oscillating ghost attractor.
The second model is a blinking piecewise-smooth Lorenz-like system which is a non-autonomous version of the system considered in [Belykh et al., 2019]. Surprising that in this case the averaged blinking system composed from two globally asymptotically stable systems has the globally stable singularly hyperbolic attractor.

\section{Blinking Nonlinear Rotator}

Here as an example we consider the equation of blinking nonlinear rotator

$$
\ddot{\varphi}+(\lambda-a \cos \varphi) \dot{\varphi}+\sin \varphi=s(t),
$$

where $\varphi$ is the phase of the rotator, $\lambda$ and $a$ are positive parameters and $s(t)$ is a randomly switching function which is chosen as $s_{1}=\gamma, s_{2}=-\gamma$ with probabilities $p_{1}=p_{2}=\frac{1}{2}$. Here the parameter $\gamma$ is a positive torque.

We consider the small switching period $\tau \ll 1$. In this case the system (5) due to (4) can be averaged over a fast time $t^{\prime}=\frac{t}{\tau}$ and as a result the random function $s(t)$ can be replaced by its average time value $\left\langle s\left(t^{\prime}\right)\right\rangle_{t^{\prime}}=$ $p_{1} \gamma-p_{2} \gamma=0$. Thus, the averaged system corresponds to the case of the autonomous system (5) with $s(t) \equiv 0$.

Consider the case of fixed values of the switching function $s(t) \equiv \gamma$. The equation (5) gets the form of the autonomous system

$$
\begin{aligned}
& \dot{\varphi}=y, \\
& \dot{y}=\gamma-\sin \varphi-(\lambda-a \cos \varphi) y .
\end{aligned}
$$

This system is invariant under involution $\varphi \rightarrow-\varphi$, $y \rightarrow-y, \gamma \rightarrow-\gamma$. Hence without loss of generality we consider only non-negative values of $\gamma$.

The partition of the parameter space $D: \gamma \geq 0, \lambda \geq$ $0, a$, whose domains correspond to different phase pictures of the system (6), was considered in [Belyustina and Belykh, 1973]. This partition for $a=1$ is depicted in Fig.1.

As far as the averaged system in our particular case is the system (6) with $\gamma=0$ we describe this case in details. Namely consider the case $\gamma=0$ and $\lambda_{h}<\lambda<a$, where $\lambda=\lambda_{h}$ is the symmetrical homoclinic bifurcation and $\lambda=a$ is Andronov-Hopf bifurcation (region III for $\gamma=0$ in Fig. 1). Increase of the parameter $\lambda$ from region I to region III at homoclinic bifurcation $\lambda=\lambda_{h}$ two stable rotating limit cycles $O_{r}^{+}$and $O_{r}^{-}$disappear and oscillating cycle $O_{c}$ is born. As a result for $\lambda_{h}<\lambda<a$ we obtain the phase picture of Fig.2(left). When $\lambda$ reaches the value $\lambda=a=1$ the stable oscillating limit cycles disappears via Andronov-Hopf bifurcation.

For $\gamma>1$ (the region VII in Fig.1) the system (6) has the globally stable rotating cycle $O_{r}^{+}$in the upper halfcylinder $y>0$ (Fig.2(right)) and for $\gamma<1$ due to the symmetry the system (6) has the globally stable rotating cycle $O_{r}^{-}$in the domain $y<0$. 
Hence, our two systems composing the blinking nonlinear rotator (5) have the globally stable cycles $O_{r}^{ \pm}$as the attractors. On the other hand the attractor $\bar{A}$ of the averaged system (6), $\gamma=0$, has the globally stable oscillating limit cycle. Hence, according to Definition $\bar{A}$ is the ghost attractor of the blinking system (5).

The interesting question on the system (5) behaviour for non-small period $\tau$ arises. In order to obtain an answer to this question we present the result of numerical simulation. In Fig. 3 the phase pictures of the blinking system (5) for three periods $\tau=0.01$ (left), $\tau=0.07$ (center) and $\tau=0.3$ (right) are presented. The globally stable limit cycle $O_{c}$ (yellow in Fig. 3) of the averaged system (the system (6) for $\gamma=0$ ) acts as a ghost attractor of the blinking system (5), and the steady trajectory of the stochastic dynamical process (blue in Fig. 3) lyes in the neighbourhood of $O_{c}$.

The size of this neighbourhood increases with increase of the period $\tau$ (see Fig. 3). First the trajectories of the blinking system fill out an annulus with respectively small divergence from the limit cycle $O_{c}$ (Fig. 3 (left)). Then the hole of the annulus disappears (Fig. 3 (center)). The further increase of the period $\tau$ leads to emergence of trajectories getting in the neighbourhood of rotating limit cycles $O_{r}^{ \pm}$(Fig. 3 (right)).

\section{Ghost Attractor of Lorenz Type}

Consider a 3-D piecewise-linear blinking system composed from subsystems $A_{s}, A_{l}$ and $A_{r}$ of the form

$$
\begin{aligned}
\dot{x} & =x, \\
A_{s}: & \dot{y}=-\alpha y, \quad(x, y, z) \in G_{s} \\
\dot{z} & =-\nu(t) z, \\
\dot{x} & =-\lambda(x+1)+\omega(z-b(t)), \quad(x, y, z) \in G_{l} \\
A_{l}: \dot{y} & =-\delta(y+1), \\
\dot{z} & =-\omega(x+1)-\lambda(z-b(t)), \\
\dot{x} & =-\lambda(x-1)-\omega(z-b(t)), \quad(x, y, z) \in G_{r} \\
A_{r}: \dot{y} & =-\delta(y-1), \\
\dot{z} & =\omega(x-1)-\lambda(z-b(t)),
\end{aligned}
$$

where $\alpha, \omega, \lambda$ and $\delta$ are positive parameters and $b(t)$, $\nu(t)$ are blinking functions. These subsystems are defined in the domains $G_{s}, G_{l}$ and $G_{r}$, respectively such that

$$
\begin{aligned}
& G_{s}:|x|<1, y \in \mathbb{R}^{1}, z<b(t), \\
& G_{l}:\left\{\begin{array}{l}
x \leq-1 \text { for } z \leq b(t) \\
x \leq-1 \text { for } z>b(t) \text { and } y \geq 0, \\
x<1 \text { for } z>b(t) \text { and } y<0,
\end{array}\right. \\
& G_{r}:\left\{\begin{array}{l}
x \geq 1 \text { for } z \leq b(t) \\
x \geq 1 \text { for } z>b(t) \text { and } y<0, \\
x>-1 \text { for } z>b(t) \text { and } y \geq 0 .
\end{array}\right.
\end{aligned}
$$

In our recent paper [Belykh et al., 2019] we introduced and described in details this system for the case of constant $b$ and $\nu$, providing a rigorous analysis of the strange attractor existence and its bifurcations. The main result of [Belykh et al., 2019], which is necessary here for studying the blinking Lorenz-type system (7), is stated in the following Theorem.

Theorem [Belykh et al., 2019]. Let the system (7) for constant parameters $\nu$ and $b$ satisfies the conditions

$$
\begin{aligned}
& \delta>\delta_{c r}=\frac{\omega \ln 2}{\pi}, \\
& b<b_{c r}=2 \sqrt{1+\frac{\lambda^{2}}{\omega^{2}}} \exp \left\{\frac{\lambda}{\omega}\left(\arctan \frac{\omega}{\lambda}+\pi\right)\right\},
\end{aligned}
$$

providing the absence of sliding motions in the absorbing domain. Then the next statements are true.

1. In the parameter regions

$$
\begin{aligned}
0<b & \leq b_{h}=\exp \frac{3 \pi \lambda}{2 \omega}, \\
b_{h}<b & <b_{h e t}=\gamma_{h e t} \exp \frac{3 \pi \lambda}{2 \omega},
\end{aligned}
$$

where $\gamma_{\text {het }}(\nu)$ is the inverse function of $\nu=1+\frac{\ln 2-\ln \gamma}{\ln (\gamma-1)}$, stable foci $e_{l}(-1,-1, b)$ and $e_{r}(1,1, b)$ are the only attractors of the system (the regions I and II in the Figure 4 , respectively).

2. In the parameter region

$$
b_{h e t} \leq b<\nu^{-1} \exp \frac{3 \pi \lambda}{2 \omega},
$$

a Lorenz-type strange chaotic attractor exists, born of a heteroclinic bifurcation for $b=b_{\text {het }}$ and coexists with two stable foci $e_{l}$ and $e_{r}$ (the region III in the Figure 4). 3. The surface

$$
b_{A H}=\nu^{-1} \exp \frac{3 \pi \lambda}{2 \omega}
$$

corresponds to Andronov-Hopf bifurcation for which two symmetrical saddle cycles stick to stable foci $e_{l}$ and $e_{r}$. 4. In the parameter region

$$
b_{A H} \leq b \leq b_{c r}
$$

a strange singular-hyperbolic attractor is the only attractor of the system (the region IV in the Figure 4).

The system (7) is the extension of original system from [Belykh et al., 2019] when parameters $b$ and $\nu$ become the next functions of time

$$
\begin{aligned}
& \nu(t)=s(t)\left(\nu_{2}-\nu_{1}\right)+\nu_{1}, \\
& b(t)=s(t)\left(b_{2}-b_{1}\right)+b_{1},
\end{aligned}
$$

where the random function $s(t) \in\{0,1\}$ has the same properties as in the Sect.3 and switches the system (7) between two systems. Namely a state $s(t)=0$ switches on the system $A\left(\nu=\nu_{1}, b=b_{1}\right)$ and the state $s(t)=1$ 

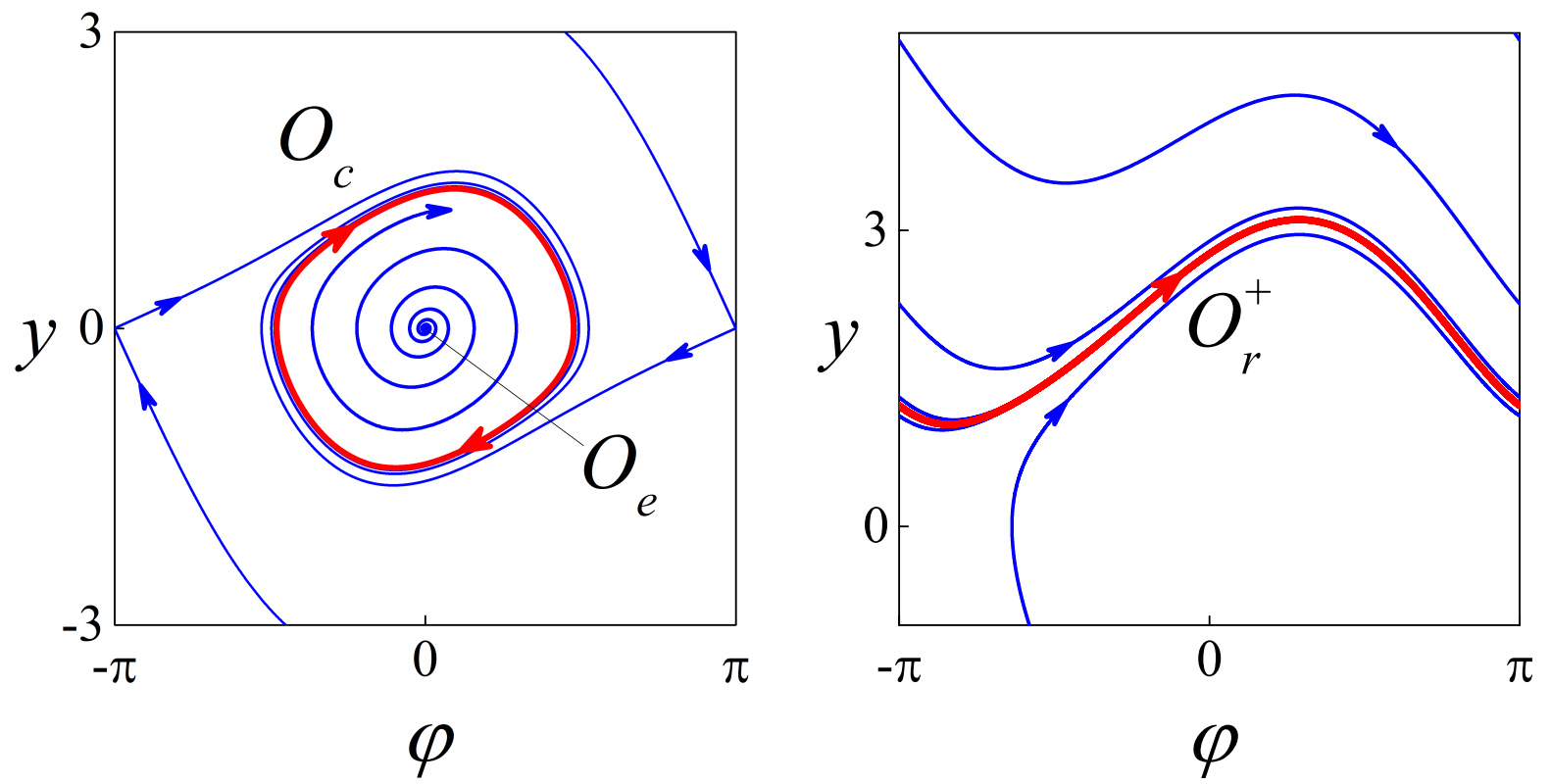

Figure 2. Phase pictures of the autonomous system (6). (Left) The globally stable oscillating cycle $O_{c}$ and the unstable equilibria $O_{e}$ for $\gamma=0$. (Right) The globally stable rotating cycle $O_{r}^{+}$for $\gamma=1.2$. Note, that for $\gamma=-1.2$, the stable rotating cycle $O_{r}^{-}$is odd symmetric to $O_{r}^{+}$and lyes in the bottom phase semi-cylinder $y<0$. The parameters $\lambda=0.75, a=1$.
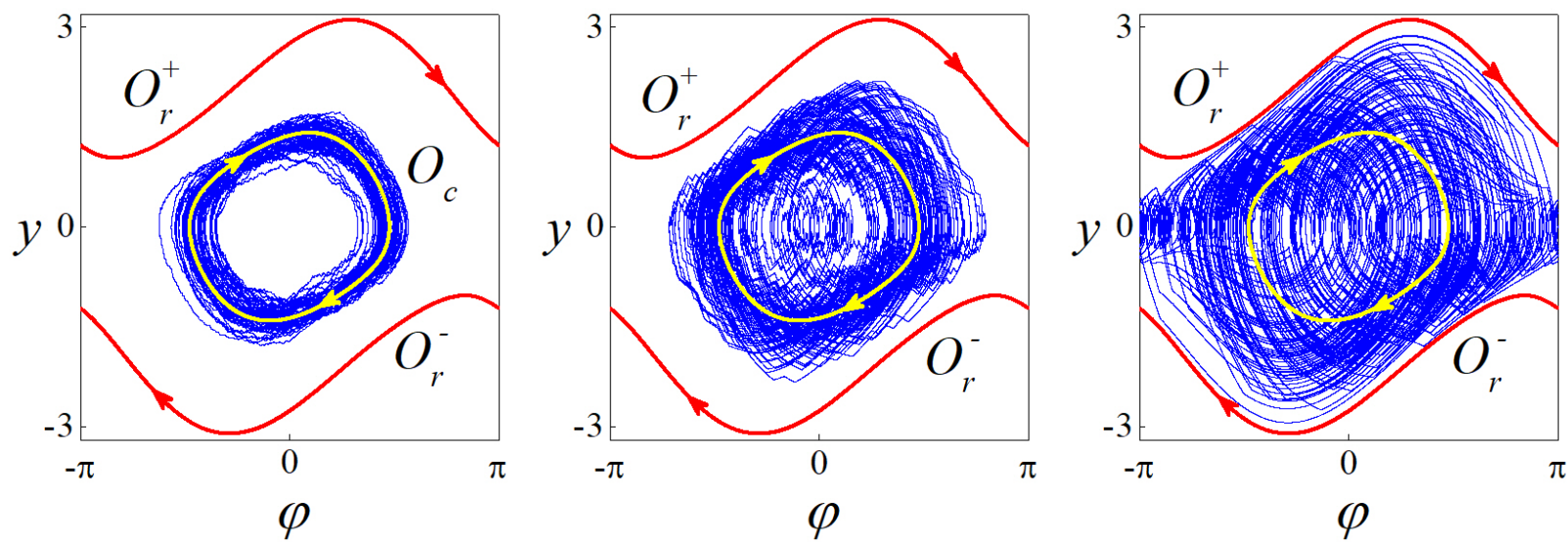

Figure 3. Non-stationary attractors of the blinking nonlinear rotator (5) (blue) for switching periods $\tau=0.01$ (left), $\tau=0.07$ (center) and $\tau=0.3$ (right). Red are the stable limit rotating cycles $O_{r}^{+}$and $O_{r}^{-}$of the composing autonomous systems $(6)$ for $\gamma=1.2$ and $\gamma=-1.2$, respectively. The stable oscillating limit cycle $O_{c}$ (yellow) is the ghost attractor. A larger switching period $\tau$ corresponds to a larger neighbourhood of the ghost attractor $O_{c}$, in which the non-stationary attracting set of the blinking system lyes. The rest parameters $\lambda=0.75$, $a=1$.

switches on the system $B\left(\nu=\nu_{2}, b=b_{2}\right)$ with equal probabilities $p_{1}=p_{2}=\frac{1}{2}$.

We define the systems $A$ and $B$ in the parameter regions II and I, respectively (see Figure 4), for which stable foci $e_{l}, e_{r}$ are the only attractors of each system.

The averaged system of blinking system (7) is obtained according to the transition from the blinking system (1) to the averaged system (4). In our case the averaged system (4) takes the form of the system (7) with constant parameters $\nu^{*}=\frac{1}{2}\left(\nu_{1}+\nu_{2}\right)$ and $b^{*}=\frac{1}{2}\left(b_{1}+b_{2}\right)$. Call- ing this averaged system $C$ we note that its parameters lye in the region IV (see Fig. 4), corresponding to the existence of the unique strange attractor. Hence we obtain the next statement.

Statement. The singular-hyperbolic attractor $\bar{A}$ of the averaged system $C$ is the ghost strange attractor of the blinking system (7). It implies that for sufficiently small switching period $\tau \ll 1$ the trajectories of the blinking system (7) are close to this ghost attractor.

To illustrate a ghost attractor of Lorenz type first we define certain systems $A(\nu=0.51, b=3.7)$ and 


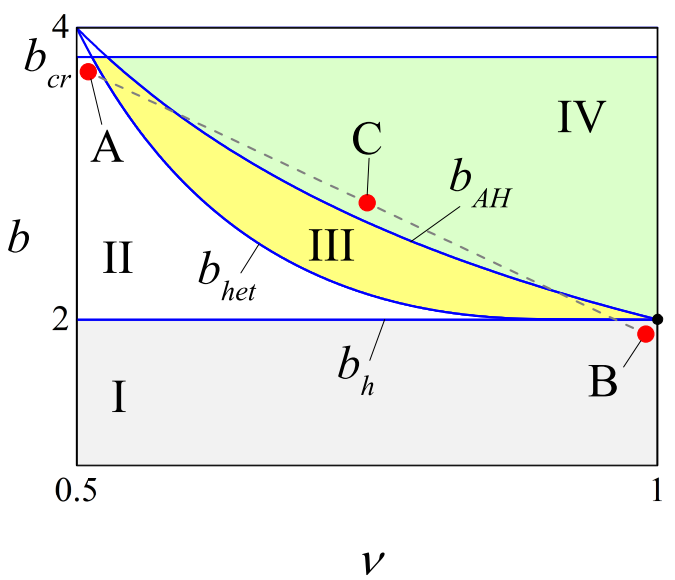

Figure 4. Bifurcation diagram of the system (7) for constant $\nu(t)=$ $\nu$ and $b(t)=b$. Regions I (gray) and II (white) correspond to globally stable foci $e_{r}$ and $e_{l}$. In region III (yellow), the system (7) has three coexisting attractors: a strange chaotic attractor and two stable foci $e_{r}$ and $e_{l}$. In region IV (green), the strange chaotic attractor is the only attractor of the system. Above the horizontal line $b_{c r}$ the conditions of the Theorem do not hold due to the appearance of sliding motions in the absorbing domain. The systems involved in blinking (A) $\nu=0.51, b=3.7$ and (B) $\nu=0.99, b=1.9$, and the averaging system (C) $\nu=0.75, b=2.8$. Parameters: $\omega=2$, $\alpha=2, \delta=0.588, \lambda=0.294$.

$B(\nu=0.99, b=1.9)$ (see Fig. 5A, 5B) from the parameter regions II and I, respectively (see Fig. 4). This systems are globally asymptotically stable having equilibria $e_{l}(-1,-1, b)$ and $e_{r}(1,1, b)$ as the attractors. Unexpectably the average system $C$ having parameters $\nu=0.75, b=2.8$ has unique Lorenz-type strange attractor (see Fig. 5C).

Similarly to the case of the blinking nonlinear rotator we present numerical results for the system (7) showing the change of the attracting set under the period $\tau$ increase (see Fig.6).

For sufficiently small switching period $\tau=0.001$ a non-stationary attractor of the blinking system (7) is close to the ghost attractor of the average system $C$ (see Fig. 6(left)).

For $\tau=0.1$ we already observe the significant change of the non-stationary attractor (see Fig. 6(center)). This change can be related to the of emergence of sliding motions in the blinking system (7).

The transition to the period $\tau=10$ (see Fig.6(right)) leads to the stabilisation of the non-stationary attractor due to the increasing impact of sliding motions [Belykh et al., 2020].

\section{Conclusions}

In this paper we presented blinking systems with instantaneous random switching between several autonomous (composing) systems in each fixed period of time. Ghost attractors as attractors of averaged systems being different from attractors of composing systems are introduced. We considered the problem of the existence of ghost attractors in the cases of blinking nonlinear rotator and three-dimensional blinking piecewise-linear system of Lorenz type. For rather fast switching using averaging approaches we found the ghost attractors for both systems. The complexity of the problem arises when switching period increases. Via numerical modeling we obtained the interval of switching period linking to zero for which non-stationary attractors of blinking systems lye in the vicinity of ghost attractors. For large switching periods in the piecewise-linear blinking system we revealed a phenomena of the attractor stabilisation due to appearance of sliding motions. Details of this stabilisation is not considered in the present paper and demands a separate study.

\section{Acknowledgements}

This work was supported by the Russian Foundation for Basic Research under Grant Nos. 18-01-00556 (to V.N.B. and N.V.B.) and 18-31-20052 (to N.V.B.) and the Russian Science Foundation (numerics) under Grant No. 19-12-00367 (to V.N.B. and N.V.B.).

\section{References}

Barabash, N. V. and Belykh, V. N. (2018a). Ghost attractor in randomly blinking phase system. In Proc. 20th Int. Scientific and Industrial Forum "Great Rivers 2018”, Nizhny Novgorod, Russia, May 15-18.

Barabash, N. V. and Belykh, V. N. (2018b). Synchronization thresholds in an ensemble of kuramoto phase oscillators with randomly blinking couplings. Radiophysics and Quantum Electronics, 60 (9), pp. 761-768.

Belykh, I., Belykh, V., Jeter, R., and Hasler, M. (2013). Multistable randomly switching oscillators: The odds of meeting a ghost. The European Physical Journal Special Topics, 222 (10), pp. 2497-2507.

Belykh, I. V., Belykh, V. N., and Hasler, M. (2004). Blinking model and synchronization in small-world networks with a time-varying coupling. Physica D: Nonlinear Phenomena, 195(1), pp. 188 - 206.

Belykh, V. N., Barabash, N. V., and Belykh, I. V. (2019). A lorenz-type attractor in a piecewise-smooth system: Rigorous results. Chaos: An Interdisciplinary Journal of Nonlinear Science, 29(10), pp. 103108.

Belykh, V. N., Barabash, N. V., and Belykh, I. V. (2020). Bifurcations of chaotic attractors in a piecewisesmooth lorenz-type system. Automation and Remote Control, (in press).

Belyustina, L. N. and Belykh, V. N. (1973). Qualitative analysis of dynamic systems in a cylinder. Differential Equations, 9(3), pp. 403-415.

Bogoliubov, N. N. and Mitropolsky, Y. A. (1966). Asymptotic methods in the theory of nonlinear oscillations. Gordon and Breach, New York.

Hasler, M., Belykh, V., and Belykh, I. (2013a). Dynamics of stochastically blinking systems. part i: Finite 

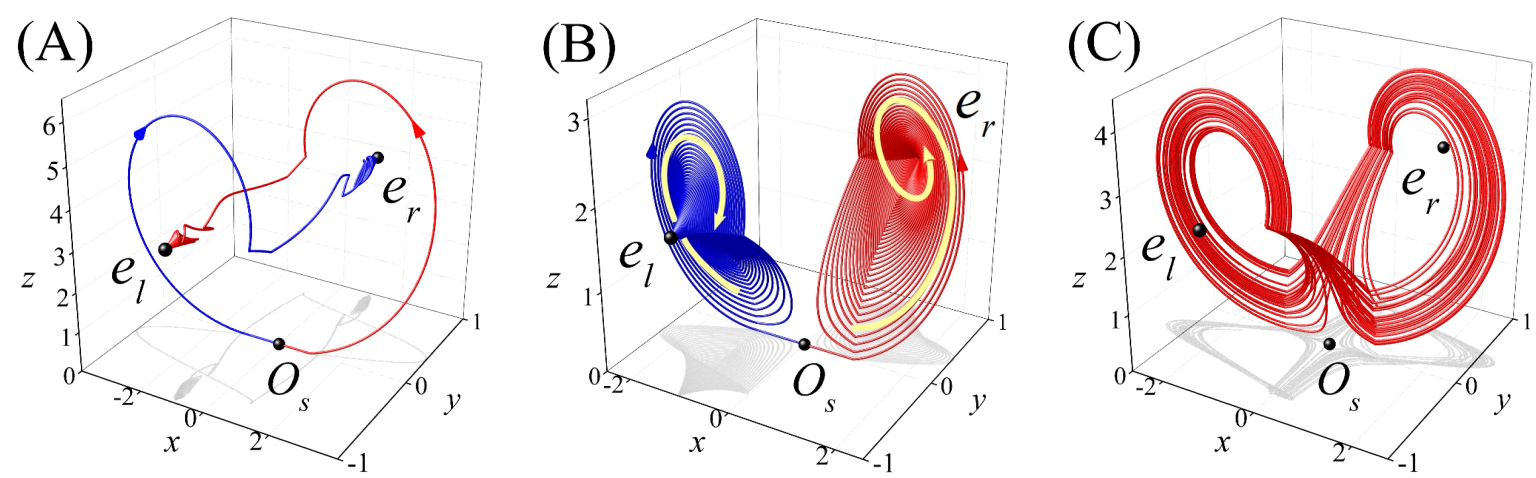

Figure 5. Phase portraits of the systems (A) and (B) involved in blinking, and of the averaged system (C). The yellow arrows in the Fig.(B) explain asymptotic stability of the foci $e_{l}$ and $e_{r}$ via dense spirals of trajectories. The parameters of the system (A) $\nu=0.51, b=3.7$, of the system (B) $\nu=0.99, b=1.9$, and of the system (C) $\nu=0.75, b=2.8$. The rest parameters: $\omega=2, \alpha=2, \delta=0.588$, $\lambda=0.294$.
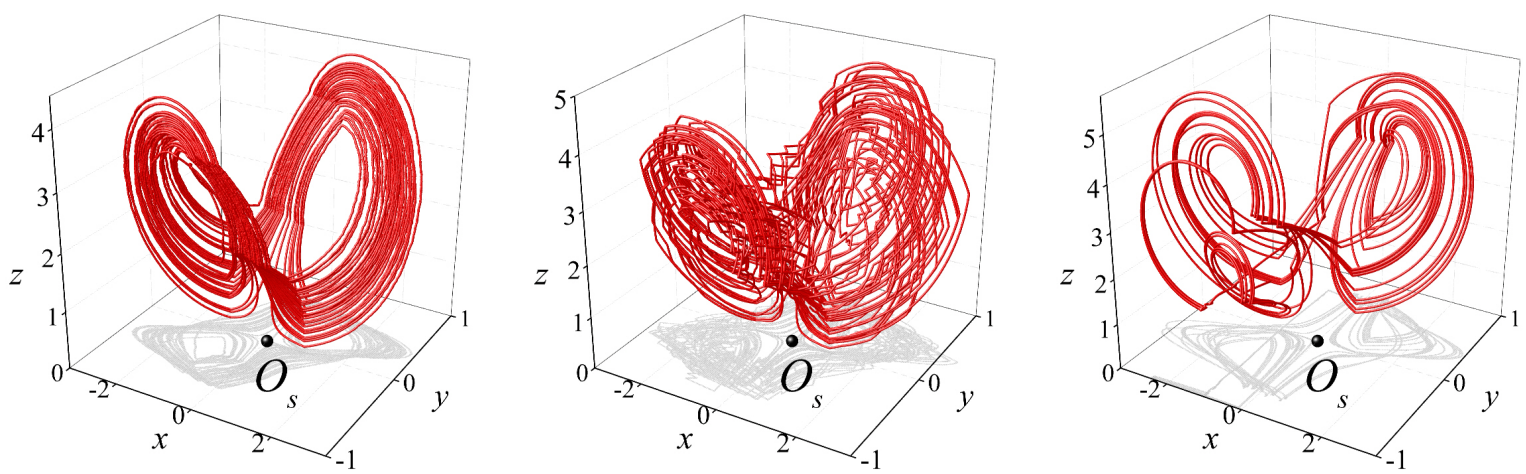

Figure 6. Trajectories of the blinking system (7) for switching periods $\tau=0.001$ (left), $\tau=0.1$ (center) and $\tau=10$ (right). Stabilisation of the trajectories for large $\tau=10$ (right) occurs via sliding motions. Parameters: $\omega=2, \alpha=2, \delta=0.588, \lambda=0.294$.

time properties. SIAM Journal on Applied Dynamical Systems, 12 (2), pp. 1007-1030.

Hasler, M., Belykh, V., and Belykh, I. (2013b). Dynamics of stochastically blinking systems. part ii: Asymptotic properties. SIAM Journal on Applied Dynamical Systems, 12 (2), pp. 1031-1084.

Khas'minskii, R. Z. (1966). A limit theorem for the solutions of differential equations with random right-hand sides. Theory of Probability \& Its Applications, 11 (3), pp. 390-406.

Kifer, Y. (2009). Large Deviations and Adiabatic Transitions for Dynamical Systems and Markov Processes in Fully Coupled Averaging. Memoirs of the American Mathematical Society. American Mathematical Society.
Mills, D. L. (1991). Internet time synchronization: the network time protocol. IEEE Transactions on Coтmunications, 39 (10), pp. 1482-1493.

Parastesh, F., Azarnoush, H., Jafari, S., Hatef, B., Perc, M., and Repnik, R. (2019). Synchronizability of two neurons with switching in the coupling. Applied Mathematics and Computation, 350, pp. 217 - 223.

Skorokhod, A. V., Hoppensteadt, F. C., and Salehi, H. (2002). Random Perturbation Methods with Applications in Science and Engineering. Applied Mathematical Sciences. Springer, New York.

Tse, C. K. and Di Bernardo, M. (2002). Complex behavior in switching power converters. Proceedings of the IEEE, 90 (5), pp. 768-781. 